\title{
Virus genotypes and responses of serum-specific antibodies in children with primary mumps and mumps reinfection
}

\author{
Rika Sakata ${ }^{1,2}$, Akira Nagita ${ }^{2}$, Minoru Kidokoro ${ }^{3}$, Atsushi Kato ${ }^{3}$ and Keiki Ogino
}

BACKGROUND: Research on children with mumps reinfection after natural infection is limited; there are currently no studies on virus-specific antibody responses in paired sera or genotyping of isolated viruses.

METHODS: This study included 281 children (147 boys and 134 girls, age: 1.2-15.9 y) with primary mumps (240), mumps reinfection after natural infection (9), mumps after previous vaccination (26), and vaccine-associated mumps (6). We measured mumps-specific serum antibodies and analyzed isolated virus genes.

RESULTS: During acute illness, series-specific IgM and IgG titers exceeded cutoff values in 240 and 232 children with primary mumps, respectively. During convalescence, IgM antibodies were positive in seven and negative in two of nine children with mumps reinfection occurring after natural infection; among 26 previously vaccinated children, 13 were positive and 13 negative. Mumps viruses were isolated from viral cultures from 42 of the 51 children. Except for 6 vaccine-associated cases, all remaining 36 cases of isolated mumps virus were identified as genotype $\mathrm{G}$.

CONCLUSION: These results suggest that measurement of IgM antibody on any day of acute illness may be indicative of primary mumps but may be inconsistent for diagnosing mumps reinfection after natural infection or previous vaccination.

M ost children with inflammatory parotid gland diseases other than mumps are treated without a known etiology. To improve etiology identification, we are attempting to provide definitive diagnoses for each child with parotid swelling; diagnosing mumps is therefore essential as a part of this process. Many children with mumps reinfection occurring after natural infection presumably are misdiagnosed in the general clinical setting as having primary mumps or other parotid diseases. To make a definitive diagnosis of reinfection, viral cultures and detection of mumps-specific IgM and IgG antibodies in paired sera are required. Although there are some reports on the response of virus-specific serum antibodies in patients with primary mumps and in those with mumps reinfection (1-7), no studies have reported the detection of specific $\operatorname{IgM}$ and $\operatorname{IgG}$ antibodies in paired sera of children with mumps reinfection occurring after natural infection. In this study, we investigated responses of both antibodies in such cases as well as in previously vaccinated children with mumps and in those with primary mumps infection. We additionally analyzed genotypes of isolated mumps viruses with viral cultures.

\section{RESULTS}

In total, 240 children were diagnosed with primary mumps. Figure 1 shows that serum-specific IgM antibody titers from days 0 to 7 of illness exceeded the cutoff value of 1.0 antibody index in all children with primary mumps at the first visit. In two children who had been evaluated on day 1 of illness, IgM antibody titers were in the intermediate range with 1.01 and 1.08 antibody indices; IgG antibody titers were 2.3 (intermediate range) and 0.7 (negative) enzyme immunoassay (EIA) titers, respectively. Both antibodies were found to be positive in both children at the second visit. In four children who had been evaluated on day 0 of illness, symptoms included fever, nausea, and poor appetite. Figure 2 shows that $\operatorname{IgG}$ antibody titers from days 0-7 exceeded the cutoff value of 2.0 EIA titers in 232 of the 240 children with primary mumps. IgG antibodies below the cutoff value were found in five children on day 1 and in three children on day 2; at the second visit, titers in all eight of these children were found to be positive. IgG antibodies in all four children who were evaluated on day 0 were positive. In children with both negative IgM and negative IgG antibodies at the first visit, neither antibody was found to be positive at the second visit. No statistical significance was found in daily changes for either antibody titer. Viral cultures were conducted in 10 children with primary mumps. Mumps viruses, all of which were identified as genotype $\mathrm{G}$, were isolated from eight children. No mumps viruses were isolated from children with both antibodies negative at the first visit.

Nine children were diagnosed with mumps reinfection after natural infection. Figure 3 shows that six children with mumps reinfection after natural infection showed negative mumps-specific IgM serum antibodies before reinfection. In five of these children, IgM antibodies were found to be positive

'Department of Public Health, Okayama University Graduate School of Medicine, Dentistry and Pharmacological Sciences, Okayama City, Okayama, Japan; ${ }^{2}$ Department of Pediatrics, Mizushima Central Hospital, Kurashiki City, Okayama, Japan; ${ }^{3}$ Department of Virology III, National Institute of Infectious Diseases, Musashimurayama City, Tokyo, Japan. Correspondence: Akira Nagita (a.nagita@mch.or.jp) 


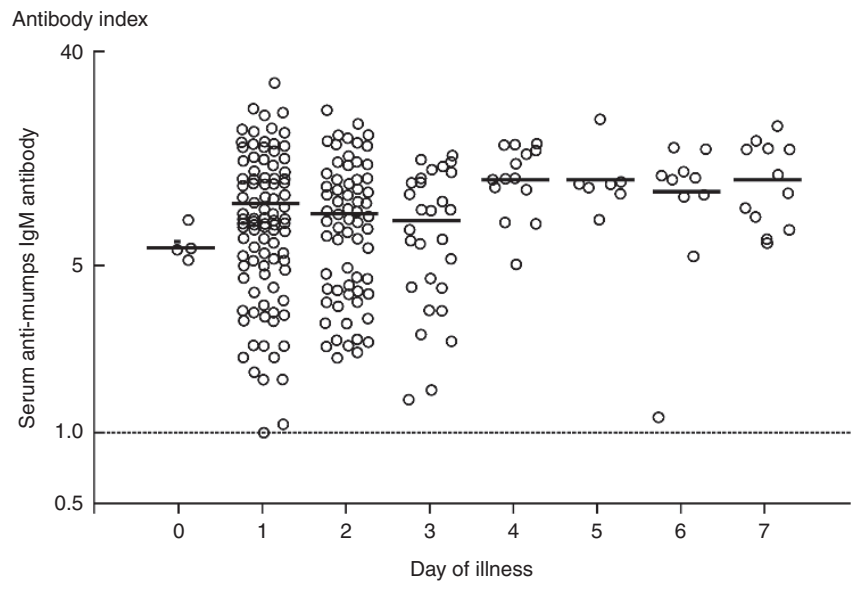

Figure 1. Serum-specific lgM antibody titers during the acute phase of illness in children with primary mumps. The $y$-axis is logarithmic. Bars indicate means, and the dotted line indicates cutoff value.

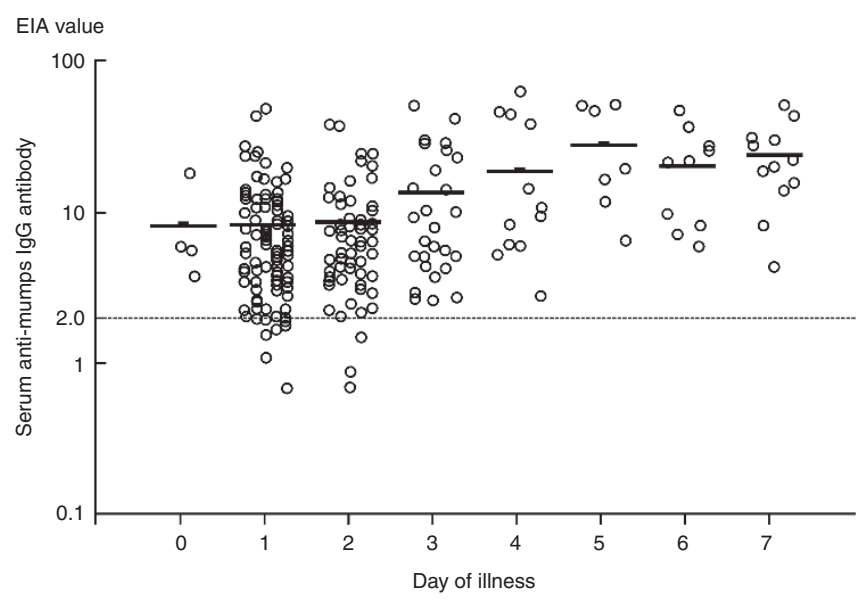

Figure 2. Serum-specific $\lg G$ antibody titers during the acute phase of illness in children with primary mumps. The $y$-axis is logarithmic. Bars indicate means, and the dotted line indicates cutoff value. EIA, enzyme immunoassay.

in the acute phase, although one of these children retained negative titers in both the acute and convalescent phases. The three remaining children with mumps reinfection after natural infection showed negative IgM antibodies in the acute phase: two of these children proceeded to develop positive titers in the convalescent phase and one retained negative titers. No difference in clinical features was found between the children with positive IgM antibodies in the convalescent phase and those with negative titers. Figure 4 illustrates that in all children with mumps reinfection after natural infection, IgG antibody titers in the convalescent phase were more than twice as high as those in the acute phase. No children with mumps reinfection after natural infection were previously diagnosed with primary mumps in our hospital. Viral cultures were conducted in all nine children, and mumps viruses, all identified as genotype G, were isolated from seven children.

Twenty-six children were diagnosed with mumps reinfection after previous vaccination, and in Table 1, we show the determination of mumps-specific IgM serum antibody in the

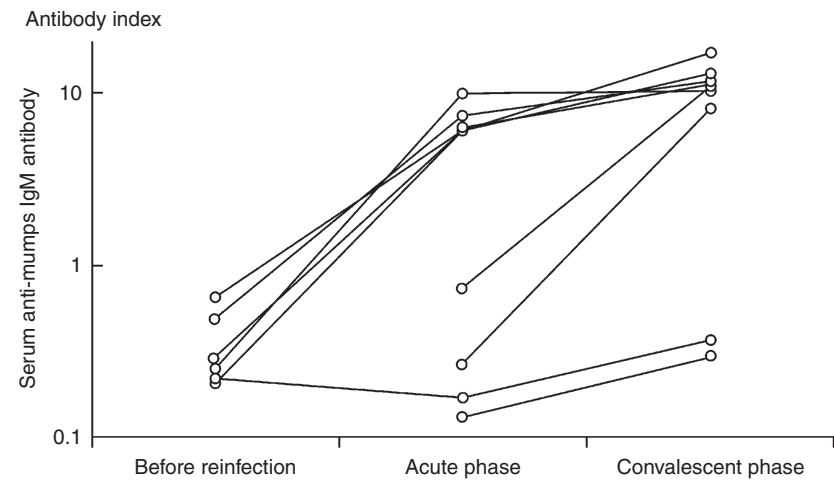

Figure 3. Serum-specific lgM antibody titers before reinfection and in the acute and convalescent phases in children with mumps reinfection after natural infection. The $y$-axis is logarithmic.

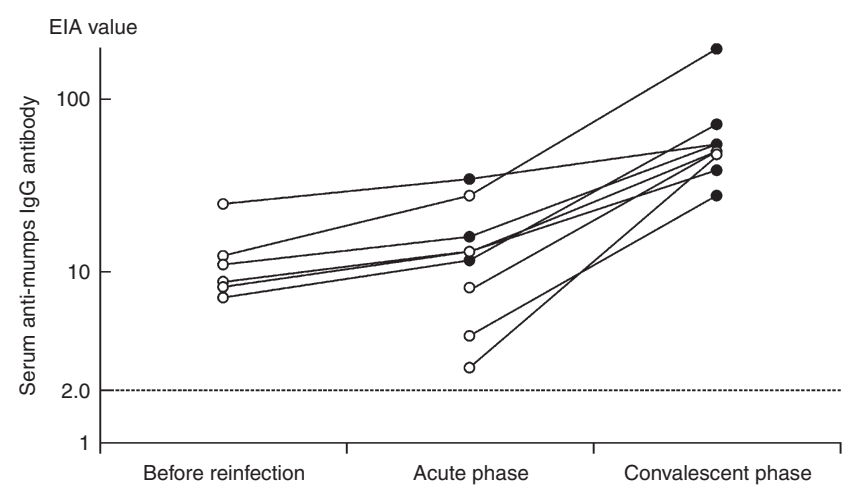

Figure 4. Serum-specific lgG antibody titers before reinfection and in the acute and convalescent phases in children with mumps reinfection after natural infection. The $y$-axis is logarithmic. The dotted line indicates cutoff value. Closed and open circles indicate specific IgM antibodies that are positive and negative, respectively. ElA, enzyme immunoassay.

Table 1. Determination of anti-mumps IgM antibodies in paired sera of children with mumps reinfection after previous vaccination

\begin{tabular}{|c|c|c|c|c|}
\hline & \multicolumn{3}{|c|}{$\begin{array}{l}\text { Serum IgM antibody } \\
\text { (acute/convalescent) }\end{array}$} & \multirow[b]{2}{*}{ Total } \\
\hline & $-1-$ & $-/+$ & $+/+$ & \\
\hline Positive virus culture only & 1 & 0 & 2 & 3 \\
\hline $\begin{array}{l}\text { Significant increase only in } \\
\text { serum } \lg G \text { antibody titer }\end{array}$ & 4 & 1 & 0 & 5 \\
\hline $\begin{array}{l}\text { Culture is positive with } \\
\text { significant increase }\end{array}$ & 8 & 4 & 6 & 18 \\
\hline Total & 13 & 5 & 8 & 26 \\
\hline
\end{tabular}

Results are shown as the number of children.

acute and convalescent phases. Twenty-three children showed significant increases in IgG antibody titers regardless of virus culture results. Twelve of these children showed negative IgM antibody results in both the acute and convalescent phases, five showed positive results only in the convalescent phase, and six showed positive results in both illness phases. Virus cultures were positive in three children who lacked significant increases in IgG antibody titers. In two of these children, IgM antibodies were positive in both phases; IgG antibody titers were 110 
and 65.2 EIA titers in the acute phase and 190 and 85.2 EIA titers in the convalescent phase. Intervals between these acuteand convalescent-phase measurements were 4 and $7 \mathrm{~d}$. The third child with a positive virus culture showed negative IgM antibody in both phases; IgG antibody titers in the acute and convalescent phases were 9.1 and 17.2 EIA titers, respectively. The interval between the two phase measurements was $4 \mathrm{~d}$. The mean \pm SD period from mumps vaccination to reinfection was $49 \pm 30$ mo (with a range of 7-147 mo and a median of $41 \mathrm{mo}$ ). Virus cultures were conducted in all 26 children, and mumps viruses, all identified as genotype $G$, were isolated from 21 children.

In six children (five boys and one girl), we observed vaccine-associated mumps, and their clinical features are shown in Table 2. The vaccine-associated Miyahara strain had been isolated after vaccination in all six cases. Ages ranged from $1 \mathrm{y}$ and 6 mo to $5 \mathrm{y}$ and $7 \mathrm{mo}$. In all children, the only symptom was salivary gland swelling that had developed 16-29 d after vaccination. Mumps-specific IgM and IgG serum antibodies were positive in all cases during the acute phase.

\section{DISCUSSION}

We considered $\mathrm{a} \geq 2$-fold increase in serum mumps-specific IgG titer in paired sera as one of the diagnostic criteria of mumps reinfection in this study based on the following findings. EIA is a reliable method to measure serum mumps-specific IgG and IgM compared with other methods (hemagglutination inhibition, complement fixation, and neutralization testing), with coefficients of variation for IgG and IgM being 12.0 and $12.4 \%$, respectively (1). The manual of the Japanese Society of Pediatric Infection recommends that a $\geq 2$-fold increase in serum mumps-specific IgG titer in paired sera is sufficient to diagnose primary mumps (8). Although sera from blood drawn at different times may vary within this range due to factors such as hydration, stress, or unrelated illness, there were no children in our study with severe conditions that would have influenced the serum IgG and IgM titers. Moreover, the US Centers for Disease Control and Prevention states that a fourfold rise in IgG titer is rarely demonstrated between paired serum samples from persons with mumps reinfection who have been vaccinated for mumps because the existing IgG will begin to rise soon after exposure and infection in vaccinated children.

Serum mumps-specific IgM antibody titers exceeded the cutoff value after day 0 in all children with primary mumps at the first visit. Although IgM antibodies in two cases were within the intermediate range, titers subsequently tested positive at the second visit. Children with negative IgM and IgG antibodies at the first visit did not have a positive antibody at the second visit. These findings suggest that measuring both serum antibodies is informative to identify causes of parotid swelling in childhood; however, IgM detection is not reliable for confirming cases of reinfection. Previous reports showed that IgM antibodies were negative in some patients with primary mumps during the acute phase. The diagnoses of primary mumps in those reports were made based on physical findings (1), significant increases in serum complement fixation test titers (2), significant increases in IgG antibody titers $(3,4)$, or positive virus cultures (2). In this study, $\operatorname{IgM}$ antibody was negative in the acute phase in more than half of children with mumps reinfection that had developed after a natural infection. These findings suggest that previously reported patients diagnosed with primary mumps who showed a negative IgM antibody in the acute phase may have had inflammatory salivary gland diseases other than mumps (1) or mumps reinfection after natural infection (1-4).

Of the nine children with reinfection after natural infection, IgM antibodies in the convalescent phase were positive in seven and negative in the remaining two. These results indicate that some children with mumps reinfection after natural infection synthesize the IgM antibody, whereas others do not. Based on these antibody responses, the diagnosis of mumps reinfection occurring after natural infection should be reconsidered, as described below. IgM antibodies in the acute phase were positive in five children; because these cases also showed positive IgG titers, the response pattern of both antibodies is the same as that in primary mumps. In such cases, a misdiagnosis of primary mumps may be made unless both antibodies were determined before reinfection. These misdiagnoses are considered to be within an acceptable error range in our etiological investigation of inflammatory parotid gland diseases, because both diagnoses accurately identify a current mumps virus infection. In four other cases in this study, however, IgM antibodies were negative in the acute phase, with two of these cases retaining negative titers throughout the convalescent phase. In investigations that determine the presence of these antibodies only in the acute phase, these four children would have been misdiagnosed, as having had a past mumps infection or an illness of unknown etiology. To avoid such misdiagnoses,

Table 2. Clinical features of children with vaccine-associated mumps (Miyahara strain)

\begin{tabular}{llllll}
\hline Case & Age & Sex & $\begin{array}{c}\text { Interval after } \\
\text { vaccination (days) }\end{array}$ & Affected organ & $\begin{array}{c}\text { IgG (EIA value)/lgM } \\
\text { (antibody index) }\end{array}$ \\
\hline 1 & 1 y $6 \mathrm{~m}$ & Male & 21 & Parotid gland & $13.6 / 3.25$ \\
2 & 1 y $8 \mathrm{~m}$ & Female & 23 & Parotid gland & $15.2 / 5.81$ \\
3 & 1 y $11 \mathrm{~m}$ & Male & 17 & Parotid gland & $2.6 / 11.11$ \\
4 & 4 y $11 \mathrm{~m}$ & Male & 29 & Parotid gland & $22.9 / 9.4$ \\
5 & $5 \mathrm{y} 1 \mathrm{~m}$ & Male & 16 & Parotid and submaxillary glands \\
6 & $5 \mathrm{y} 7 \mathrm{~m}$ & Male & 18 & Parotid gland & $16.7 / 9.63$ \\
\hline
\end{tabular}

ElA, enzyme immunoassay. 
we are attempting to determine etiology by appropriately performing parotid ultrasonography and smear microscopy of parotid saliva; when necessary, viral cultures are additionally conducted in the acute phase, and both antibodies are measured in the convalescent phase. To date, it appears that there is only one report of serum antibody response in patients with mumps reinfection after natural infection. Gut et al. (7) investigated various characteristics of such patients diagnosed with reinfection based on both a significant rise in IgG antibody titers and negative IgM antibody titers throughout the course of illness. The clinical features of these patients and their antibody responses were compared with those in other patients with primary mumps who manifested positive IgM titers. The study was retrospective, so patients with mumps reinfection after natural infection showing positive IgM antibody were not enrolled as subjects, and only a few pediatric patients were included in the study. The methods used in our study resolve such disadvantages.

When children previously vaccinated for mumps presented with parotid swelling, we conducted the aforementioned examinations while also considering the differential diagnoses of mumps reinfection and other parotid diseases. Of the 26 previously vaccinated children with mumps reinfection, mumps-specific IgM serum antibody was positive in the acute phase for 8 cases and negative for 18 cases. These results indicate that some previously vaccinated children with mumps reinfection also synthesize the IgM antibody, whereas others do not. The mean period of $49 \mathrm{mo}$ from mumps vaccination to reinfection was considerably long, and our results may simply reflect that the longer the time interval from vaccination, the greater is the cumulative opportunity for exposure (5). In three previously vaccinated children with mumps reinfection who had been diagnosed by positive viral cultures alone, acuteand convalescent-phase IgG titers increased. The intervals of paired serum sampling for these children were $\leq 7 \mathrm{~d}$. It takes at least 2 wk to obtain viral culture results. Based on these findings, to diagnose as soon as possible, IgG antibodies should be measured $>7 \mathrm{~d}$ after acute-phase measurements or real-time PCR assays should be introduced (9).

In children with vaccine-associated mumps, the intervals from vaccination to illness onset ranged from 16-29 d. The incubation period for mumps is $2-3 \mathrm{wk}$. Secondary diseases such as swelling of otherwise healthy salivary glands and meningitis sometimes develop after the onset. The shortest interval of $16 \mathrm{~d}$ is consistent with the length of the incubation period, and the longest interval of $29 \mathrm{~d}$ may reflect the onset period of secondary diseases.

We conducted viral cultures to detect pathogens in viral parotitis, including those of mumps reinfection. Genotyping of isolated mumps viruses was also conducted to investigate locally prevalent strains in addition to genotype differences between primary mumps and reinfection. Results showed all viruses of both primary mumps and reinfection to be genotype $\mathrm{G}$ viruses. The G-type mumps virus has been prevalent in Japan since 2000 (ref. 10). To the best of our knowledge, previous reports have not identified genotypes in mumps reinfections occurring after natural infection. These findings suggest that the G-type mumps virus has recently prevailed in both primary mumps and reinfection in Japan.

\section{METHODS \\ Subjects}

In total, 437 children visited our hospital with salivary gland swelling over a 9-y period between December 2004 and November 2013. Of these, 281 children demonstrated one or more of the following results, qualifying them for inclusion in this study: positive serum mumpsspecific IgM antibody, a significant increase in serum IgG antibody titers, or a positive mumps viral culture. There were 147 boys and 134 girls, with ages ranging from 1.2 to $15.9 \mathrm{y}$ (mean \pm SD: $5.2 \pm 2.0 \mathrm{y}$ ). Among children with primary mumps, 12 developed only submandibular gland swelling, and three were secondarily associated with meningitis.

\section{Diagnoses of Primary Mumps and Reinfections}

A diagnosis of primary mumps was made with the detection of mumps-specific IgM serum antibody in the acute phase (defined as the time period when symptoms derived from mumps were found) in children with no previous history of mumps infection or vaccination. Despite detection of positive IgM, children diagnosed with mumps reinfection according to the diagnostic criteria described below were excluded from the group of primary mumps. A diagnosis of mumps reinfection after previous natural infection was made with $a \geq 2$-fold increase in mumps-specific IgG titers from paired sera or the isolation of wild strains, or both, in children without previous vaccination who showed both negative mumps-specific IgM and positive IgG antibodies in serum before the onset of salivary gland swelling or in the acute phase. A diagnosis of mumps reinfection after previous vaccination was made with a $\geq 2$-fold increase in mumps-specific IgG titers from paired sera or the isolation of wild strains, or both, in previously vaccinated children. A diagnosis of vaccine-associated mumps was made with the isolation of a vaccine strain (Miyahara strain) in children with postvaccinal swelling of the salivary glands.

\section{Determination of Mumps Antibodies and Genotypes}

Only clinical features related to inflammation of the salivary glands, such as salivary gland pain and swelling, were considered mumpsderived symptoms. The first day of the symptoms was recorded as day 1 of illness. Serum-specific IgM and IgG antibodies in children with primary mumps were measured from day 0 , the day before symptoms first appeared, to day 7 of the illness. Another serum sample was obtained at the second visit, more than $3 \mathrm{~d}$ after the first visit. When IgM antibody at the first visit was determined to be in the intermediate range, both antibodies were remeasured at the second visit. When the IgM antibody at the first visit was positive and the IgG antibody at the first visit was negative or was determined to be in the intermediate range, IgG antibody was remeasured at the second visit. When IgM antibody was negative and ultrasonography suggested viral parotitis at the first visit, both antibodies were remeasured at the second visit. IgM and IgG antibodies in children with mumps reinfection after natural infection were measured at the first visit, within $2 \mathrm{~d}$ after onset of symptoms, and at the second or third visit, more than $3 \mathrm{~d}$ after the first visit.

When pre-onset preserved serum had been obtained, both antibodies were measured. In our hospital, sera of children who had undergone blood examinations were stored for at least $6 \mathrm{mo}$. When both antibodies had been measured before onset, the data were also investigated as pre-onset serum antibody titers. Serum mumps-specific IgG and IgM antibodies were measured in each child with parotid swelling over $10 \mathrm{y}$ for a study on the etiology of inflammatory parotid gland diseases. In previously vaccinated children with mumps reinfection, both antibodies were measured at the first visit, within $2 \mathrm{~d}$ after onset, and at the second or third visit, more than $3 \mathrm{~d}$ after the first visit. Both antibodies in children with suspected vaccine-associated mumps were measured at the first visit within $2 \mathrm{~d}$ after onset. When either mumps reinfection or another viral infection was suspected, ultrasonography was performed, buccal swabs at the first visit were sampled, and virus cultures were 


\section{Articles | Sakata et al.}

conducted. IgM and IgG antibodies were determined using commercially available measurement kits (mumps virus antibody EIA Seiken IgM and IgG; Denka Seiken, Tokyo, Japan). The cutoff value of IgM antibody was set at 1.0 antibody index, which was the mean $+6 \mathrm{SD}$ value of the titers in healthy individuals. The cutoff value of the IgG antibody was set at 2.0 EIA titers, which was the mean $+3 \mathrm{SD}$ value of the titers in subjects showing negative complement fixation tests. Taking into account the measurement error of each kit, the intermediate ranges of the IgM and IgG antibodies were determined to be 0.80 1.20 antibody index and 2.0-3.9 EIA titers, respectively. Values below the range were considered negative, and those above the range were considered positive. Virus cultures were conducted by growing viruses using cell culture after having preserved samples in phosphate buffer solution containing albumin and gentamicin. RNA of isolated viruses was extracted, and the regions of 728 bases (bases 6076-6803 on viral genomes), including the $S H$ gene, were amplified. The base sequence of the gene obtained after amplification was analyzed phylogenetically and was genotyped according to the classification criteria recommended by the World Health Organization LabNet meeting (11).

\section{Ethical Considerations and Statistical Analyses}

This study was approved by the Institutional Review Board of Mizushima Central Hospital. Measurements of mumps-specific IgM and IgG serum antibodies, virus cultures, and genotyping were conducted after obtaining consent from the subjects' parents. Antibody titers are represented on the $y$-axis of the figures as common logarithms, because they were measured after sera were diluted 1/200. Quantitative results are expressed as mean $\pm \mathrm{SD}$. Regression analysis was used to analyze daily changes in IgM and IgG antibody titers in children with primary mumps. Statistical significance was defined as $P<0.05$.

\section{STATEMENT OF FINANCIAL SUPPORT}

None received.

Disclosure: The authors have no potential or perceived conflicts of interest to report with respect to this article.

\section{REFERENCES}

1. Ukkonen P, Granström ML, Penttinen K. Mumps-specific immunoglobulin $M$ and $G$ antibodies in natural mumps infection as measured by enzyme-linked immunosorbent assay. J Med Virol 1981;8: $131-42$.

2. Gut JP, Spiess C, Schmitt S, Kirn A. Rapid diagnosis of acute mumps infection by a direct immunoglobulin $\mathrm{M}$ antibody capture enzyme immunoassay with labeled antigen. J Clin Microbiol 1985;21:346-52.

3. Nicolai-Scholten ME, Ziegelmaier R, Behrens F, Höpken W. The enzymelinked immunosorbent assay (ELISA) for determination of IgG and IgM antibodies after infection with mumps virus. Med Microbiol Immunol 1980;168:81-90.

4. Sakata H, Tsurudome M, Hishiyama M, Ito Y, Sugiura A. Enzyme-linked immunosorbent assay for mumps IgM antibody: comparison of IgM capture and indirect IgM assay. J Virol Methods 1985;12:303-11.

5. Briss PA, Fehrs LJ, Parker RA, et al. Sustained transmission of mumps in a highly vaccinated population: assessment of primary vaccine failure and waning vaccine-induced immunity. J Infect Dis 1994;169:77-82.

6. Rota JS, Turner JC, Yost-Daljev MK, et al. Investigation of a mumps outbreak among university students with two measles-mumps-rubella (MMR) vaccinations, Virginia, September-December 2006. J Med Virol 2009;81:1819-25.

7. Gut JP, Lablache C, Behr S, Kirn A. Symptomatic mumps virus reinfections. J Med Virol 1995;45:17-23.

8. Ihara T. Mumps virus [Munpusu uirusu]. In: Japan Pediatric Infectious Diseases Society, eds. Pediatric Infectious Manual [Shouni-kansensyou Manyuaru] 2012. Tokyo, Japan: Tokyo Igakusha Ltd., 2011:304-11.

9. Jin L, Feng Y, Parry R, Cui A, Lu Y. Real-time PCR and its application to mumps rapid diagnosis. J Med Virol 2007;79:1761-7.

10. Momoki TS. Genotyping of mumps virus detected in Yokohama City from 1999 to 2010. Jpn J Infect Dis 2013;66:226-31.

11. World Health Organization. Mumps virus nomenclature update: 2012. Wkly Epidemiol Rec 2012;87:217-24. 